\title{
Self-Care Activities among Patients with Type 2 Diabetes in Morocco: Prevalence and Determinants
}

\author{
Latifa Adarmouch ${ }^{1,2}{ }^{*}$, Majda Sebbani ${ }^{1,2}$, Latifeh Dahmash ${ }^{1}$, Mohamed Amine ${ }^{1,2}$ \\ ${ }^{1}$ Clinical Research Unit, Mohammed VI University Hospital, Marrakech, Morocco \\ ${ }^{2}$ Community Medicine and Public Health Department, PCIME Laboratory, School of Medicine, Cadi Ayyad University, \\ Marrakesh, Morocco
}

Email: ^l.adarmouch@hotmail.com

How to cite this paper: Adarmouch, L., Sebbani, M., Dahmash, L. and Amine, M. (2020) Self-Care Activities among Patients with Type 2 Diabetes in Morocco: Prevalence and Determinants. Open Journal of Epidemiology, 10, 55-65.

https://doi.org/10.4236/ojepi.2020.101005

Received: December 10, 2019

Accepted: January 28, 2020

Published: January 31,2020

Copyright $\odot 2020$ by author(s) and Scientific Research Publishing Inc. This work is licensed under the Creative Commons Attribution International License (CC BY 4.0).

http://creativecommons.org/licenses/by/4.0/

(c) (i) Open Access

\begin{abstract}
Background: Self-care is an important, though often neglected, area of type 2 diabetes management in lower and middle income countries (LMICs). In Morocco, whilst the evolution of the disease is increasing rapidly, evidence documenting disease self-care patterns remains scarce. Objectives: To estimate the prevalence of self-care activities among patients with type 2 diabetes in Morocco, and to identify the factors associated with good self-care practices. Methods: We performed a secondary analysis of data of 406 patients aged 30 years old and over, diagnosed with type 2 diabetes mellitus for at least 6 months. Self-care activities were assessed using the Moroccan version of the Summary of diabetes self-care activities. Studied factors included socio-demographics, disease features and healthcare use. Unadjusted and adjusted odds ratios were calculated using logistic regression. Results: Mean age was $55.8 \pm 11.6$ years old. Females represented $68.7 \%$ of the respondents. Mean estimates of the frequency of self-care practices exceeded 3.5 days per week for diet, exercise and foot-care; of these, good dietary behavior was the most prevalent (63.6\%). In multivariate analysis, females displayed better dietary behavior $(\mathrm{OR}=1.81[1.27-2.58])$, and less frequent foot care $(\mathrm{OR}=1.81[1.27-2.58])$ than males. Lower levels of exercise were associated with being female $(0.42[0.26-0.68])$, and belonging to the higher income category (0.55 [0.34 - 0.88]). Residents in rural areas also reported better exercise practices $(1.72[1.07-2.78])$. Conclusion: This study draws attention to self-care practices and their determinants in the Moroccan context. Such findings should help in the design, implementation, and evaluation of self-management interventions for people with type 2 diabetes.
\end{abstract}

\section{Keywords}

Self-Care, Self-Management, Diabetes Mellitus, Type 2, Determinants, 
Morocco

\section{Introduction}

Globally, the burden of disease is undergoing an epidemiologic shift from communicable to non-communicable disease conditions. Of these, Diabetes Mellitus $(\mathrm{DM})$ is considered the 8th leading cause of death: recent pooled global estimates show a prevalence of $8.5 \%$ amongst adults [1], an almost 2 fold increase in the last 30 years. Across the African continent, the estimated number of those living with the disease has risen exponentially in the same time frame; from an estimated 4 million in 1980 to 25 million in 2014. Within Morocco, similar trends and concerns are observed due to both the changing lifestyle and demographic make-up of the population (an associated trend with rapid urbanization). Lower and middle income countries (LMICs) experiencing this transition, however, are often disproportionately affected by the condition, whilst simultaneously lacking sufficient resources to tackle it [2] [3] [4].

Self-management by the patient constitutes a cornerstone area in chronic disease care. It focuses on knowledge and self-care skills, encouraging patients to take an active role in shaping and managing their treatment regimen. For diabetes, self-management education interventions have been shown to be effective in improving glycemic control, reducing the onset of complications and improving quality of life [5] [6]. Core self-care practices for patients with diabetes include diet, physical activity, foot-care, blood sugar monitoring and treatment adherence. Alongside assessment of the levels of self-care behaviors, understanding their determinants is important to enable health professionals to design appropriate interventions. Factors that may affect self-management include demographic, socioeconomic, psychological, health status and the health-care system [7] [8].

In the Moroccan setting, there is very little evidence assessing the degree to which patients with diabetes mellitus carry out self-care activities, though published studies have reported poor health outcomes among this population [9]. In a recent study, Chadli et al. reported low achievement of the international target of $\mathrm{HbAc1}<7 \%$, alongside low adherence to self-monitoring of blood glucose [10]. For those reasons, assessing self-care practices and their determinants in this context represents an important area of investigation with the aim of developing effective strategies to improve diabetes self-management.

Therefore, the objectives of this study were to estimate the prevalence of self-care activities among patients with type 2 diabetes in Morocco, and to identify the factors associated with good self-care practices.

\section{Material and Methods}

For this secondary analysis, we pooled data from three separate studies that were 
focused upon patient-reported outcomes among diabetic patients. These studies were carried out in different regions in Southern Morocco within a 3-year period (from 2012-2014). Inclusion criteria were: patients with type 2 diabetes, over 30 years of age, and having been diagnosed for 6 months or more. Volunteers were recruited through patient organizations and primary health care facilities. Trained final year medical students or graduates interviewed the participants after having obtained their verbal consent.

Data were collected using a structured questionnaire developed by the research team, which captured data on several domains. Socio-demographics included age, gender, marital status, educational level, professional activity, residence (rural/urban) and monthly income. We also recorded disease duration, insulin therapy and previous hospitalization related to diabetes or its complications. Finally, data concerning access to care included: medical insurance, a medical visit during the last month and the number of medical visits during the past year.

Self-care activities were assessed using the Summary of Diabetes Self-Care Activities (SDCSA) [11]. This tool consists of 10 items that evaluate diet (general and specific), exercise, blood sugar testing and foot-care on a weekly frequency scale (scores ranging from 0 - 7 referring to the number of days a specific behavior was performed during the past week). The scale has been translated and adapted into many languages and used in various settings including a proposed version for use in the Moroccan context [12]. The Moroccan version consists of 8 items instead of 10, having excluded items 3 and 4 that focused on areas of specific diet (fruit and vegetable servings and consumption of high fat products) because of difficulties during the process of cross-cultural adaptation and low psychometric performances. We used the Moroccan version of the scale: items with corresponding dimensions are presented in Table 1.

Table 1. Items and dimensions of the Moroccan version of the summary of diabetes self-care activities (SDSCA).

\begin{tabular}{cl}
\hline Dimensions & \multicolumn{1}{c}{ Items } \\
\hline General diet $\quad$ How many of the last seven days have you followed a healthful eating plan? \\
On average, over the past month, how many days per week have you followed \\
your eating plan? \\
On how many of the last seven days did you participate in at least 30 minutes \\
of physical activity? (Total minutes of continuous activity, including walking)? \\
$\begin{array}{l}\text { On how many of the last seven days did you participate in a specific exercise } \\
\text { session (such as swimming, walking, biking) other than what you do around } \\
\text { the house or as part of your work? }\end{array}$ \\
Blood sugar testing
\end{tabular}


Statistical analysis was performed using SPSS version 16.0 for Windows. Qualitative variables were described using frequencies and quantitative variables were described using means and standard deviations. Additionally, medians were used to describe scores of dimensions of the SDSCA. The score of each dimension of the SDSCA was computed as the mean of the ratings of the two constituting items. Good self-care practices were defined using a threshold of 5 days/week for each dimension. Prevalence and 95\% confidence intervals were calculated for good dietary, exercise, blood sugar monitoring and foot-care behaviors. The odds ratios for sociodemographic factors, disease features and other variables were calculated in univariate analysis. Binary logistic regression was used to calculate adjusted odds ratios. For each dimension of good self-care practices a multivariate model was tested including all candidate variables that were significant at 0.10 in univariate analysis, and adopting a Stepwise approach. The level of significance was set at $5 \%$.

\section{Results}

We analyzed data from 406 eligible individuals. Their mean age was $55.8 \pm 11.6$, with values ranging from 30 to 98 years old. Females represented $68.7 \%$ of the respondents. The study sample was mostly from low socioeconomic group: a majority was illiterate, less than one third had a professional activity and 58.5\% reported less than 150 US\$ as monthly household income. Mean disease duration was $7.6 \pm 6.1$ years, ranging from 6 months to 30 years. Insulin treatment was reported by $35 \%$ of participants. Table 2 reports the description of the participants' characteristics.

Self-care frequency means exceeded 3.5 days per week for diet, exercise and foot-care. Observing diet was the most frequently reported self-care practice, while blood sugar monitoring was the least observed. Similar findings are reported

Table 2. Description of the participants' characteristics.

\begin{tabular}{ccc}
\hline Characteristic & $\mathrm{N}$ & $\%$ \\
Female $(\mathrm{n}=406)$ & 279 & 68.7 \\
Age $\geq 55$ years old $(\mathrm{n}=406)$ & 221 & 54.4 \\
Married $(404)$ & 294 & 72.8 \\
Rural $(\mathrm{n}=406)$ & 255 & 62.8 \\
Illiterate $(\mathrm{n}=403)$ & 291 & 71.7 \\
Professional activity $(\mathrm{n}=398)$ & 124 & 31.2 \\
Health insurance $(\mathrm{n}=397)$ & 144 & 58.5 \\
Monthly household income $<150 \mathrm{US} \$(\mathrm{n}=246)$ & 170 & 42.8 \\
Disease duration $\geq 5$ years $(\mathrm{n}=398)$ & 239 & 60.1 \\
Insulin $(\mathrm{n}=406)$ & 142 & 35.0 \\
Previous hospitalization related to diabetes $(\mathrm{n}=274)$ \\
$\geq 3$ medical visits in the last year $(\mathrm{n}=371)$
\end{tabular}


when we considered the prevalence of good self-care behaviors. Good dietary behavior was the most prevalent (63.6\%). Good blood sugar monitoring behavior was reported by 15 individuals (Table 3 ).

In univariate analysis (Table 4), females, older individuals, illiterate people and those residing in rural areas reported better diet behavior. Married individuals, those engaged in professional activity, and those with medical insurance were less likely to report good dietary practices.

Participants living in rural areas reported better exercise behaviors compared to those in urban areas, and women tended to report less exercise than men. With regard to good blood sugar monitoring behavior, illiterate participants reported lower frequency while those of the higher economic class reported higher frequency. Good foot-care practice was positively associated with a previous hospital admission related to diabetes, a medical visit during the last month and 3 or more medical visits over the past year.

In multivariate analysis (Table 5), women presented better dietary behavior,

Table 3. Description of self-care practices.

\begin{tabular}{ccccccc}
\hline Dimension & \multicolumn{3}{c}{ Scores of self-care dimensions } & \multicolumn{2}{c}{$\begin{array}{c}\text { Prevalence of good self-care } \\
\text { behaviors ( } \geq 5 \text { days/week })\end{array}$} \\
\hline & Mean & $\begin{array}{c}\text { Standard } \\
\text { deviation }\end{array}$ & Median & N & $\%$ & $\begin{array}{c}95 \% \text { confidence } \\
\text { interval }\end{array}$ \\
\hline Diet & 4.95 & 2.63 & 7.0 & 257 & 63.6 & $58.7-68.3$ \\
Exercise & 3.76 & 2.74 & 3.5 & 158 & 38.9 & $34.1-43.8$ \\
Blood sugar monitoring & 0.81 & 1.42 & 0.0 & 15 & 3.7 & $2.1-6.0$ \\
Foot-care & 3.79 & 2.92 & 3.5 & 172 & 42.5 & $37.6-47.4$ \\
\hline
\end{tabular}

Table 4. Univariate analysis of factors associated to good self-care behaviors: unadjusted odds ratios (OR and $95 \%$ confidence intervals).

\begin{tabular}{|c|c|c|c|c|}
\hline Variables & Good dietary behavior & Good exercise behavior & $\begin{array}{l}\text { Good blood sugar } \\
\text { monitoring behavior }\end{array}$ & $\begin{array}{c}\text { Good } \\
\text { foot-care behavior }\end{array}$ \\
\hline Female & $1.86(1.21-2.87)$ & $0.52(0.34-0.80)$ & $0.47(0.24-1.94)$ & $0.65(0.43-1.00)$ \\
\hline Age $\geq 55$ years old & $1.65(1.10-2.49)$ & $1.39(0.94-2.09)$ & $0.55(0.99-1.57)$ & $1.31(0.88-1.95)$ \\
\hline Married & $0.51(0.31-0.84)$ & $1.31(0.83-2.06$ & $1.02(0.32-3.27)$ & $1.15(0.74-1.79)$ \\
\hline Rural area & $1.87(1.32-2.84)$ & $1.55(1.02-2.37)$ & $0.88(0.31-2.52)$ & $0.79(0.53-1.19)$ \\
\hline Illiterate & $1.84(1.18-2.87)$ & $1.40(0.89-2.18)$ & $0.24(0.08-0.69)$ & $0.87(0.56-1.36)$ \\
\hline Professional activity & $0.54(0.35-0.84)$ & $1.41(0.92-2.17)$ & $2.62(0.93-7.39)$ & $1.39(0.90-2.18)$ \\
\hline Monthly income $\geq 150$ US\$ & $0.65(0.93-1.09)$ & $0.63(0.37-1.08)$ & $8.93(1.06-75.41)$ & $1.48(0.88-2.44)$ \\
\hline Medical insurance & $0.61(0.40-0.93)$ & $0.74(0.49-1.11)$ & $1.83(0.62-5.38)$ & $1.24(0.83-1.85)$ \\
\hline Disease duration $\geq 5$ years & $1.19(0.78-1.79)$ & $0.92(0.61-1.39)$ & $0.57(0.20-1.61)$ & $1.21(0.81-1.82)$ \\
\hline Insulin & $0.73(0.48-1.12)$ & $0.82(0.54-1.25)$ & $1.67(0.59-4.71)$ & $1.47(0.97-2.22)$ \\
\hline Previous hospitalization due to diabetes & $0.67(0.39-1.16)$ & $0.68(0.39-1.18)$ & $2.34(0.76-7.20)$ & $1.89(1.10-3.25)$ \\
\hline Three medical visits or more during the past year & $1.01(0.66-1.55)$ & $0.99(0.65-1.50)$ & $2.72(0.82-9.00)$ & $1.87(1.23-2.84)$ \\
\hline One medical visit during the last month & $1.12(0.73-1.73)$ & $1.44(0.95-2.20)$ & $1.35(0.47-3.89)$ & $1.58(1.04-2.41)$ \\
\hline
\end{tabular}


Table 5. Multivariate analysis of factors associated with good self-care behaviors: adjusted odds ratios (OR and 95\% confidence intervals).

\begin{tabular}{cccc}
\hline Variables & $\begin{array}{c}\text { Good } \\
\text { dietary behavior }\end{array}$ & $\begin{array}{c}\text { Good } \\
\text { exercise behavior }\end{array}$ & $\begin{array}{c}\text { Good } \\
\text { foot care behavior }\end{array}$ \\
\hline Female & $1.81(1.27-2.58)$ & $0.42(0.26-0.68)$ & $0.54(0.39-0.73)$ \\
Rural area & - & $1.72(1.07-2.78)$ & - \\
Monthly income $\geq 150$ US\$ & - & $0.55(0.34-0.88)$ & - \\
\hline
\end{tabular}

and lower foot care frequency than men. Lower levels of exercise were associated with being female, residing in urban areas and belonging to higher income category. Logistic regression was not performed for good blood sugar monitoring behavior due to the small number of respondents in this category $(n=15)$.

\section{Discussion}

This study was conducted in order to describe the prevalence of self-care practices among type 2 diabetic patients in the Moroccan context, and to explore the factors associated with those practices. Such data are important to understand patterns and specificities of self-care among the diabetic population here, and to provide a stronger evidence-base to guide the development of future interventions.

Overall, diet was the most frequently observed behavior within the present study, followed by foot-care practices (42.5\%). BSL was the least observed behavior, consistent with findings reported in previous literature [13] [14]. Responses related to exercise activities were also markedly low in this cohort, with less than $2 / 5$ of participants reporting good practice. We stress on the fact that low self-care levels constitute a serious problem in LMICs as shown in previous literature [15] [16] [17] [18] [19]. Findings from this study emphasize a similar situation in this context, and highlights the need for tailored interventions to more definitively address this.

Demographic factors associated with good dietary practices in univariate analysis were being female and within the older age group ( $>55$ years of age), being illiterate, and living in rural areas. Sex has also been shown to be associated with dietary practices in other studies, with females generally showing higher rates of compliance [20] [21]. Age has been shown elsewhere as being a positive predictor of good dietary self-care, which has been suggested to be a result of better disease management practices over time. For instance, Rajasekharan et al. found that patients who had diabetes for $>10$ years had better eating plans, and less high fat food within their diet [17]. Married patients had lower scores for good diet outcomes in this analysis. Though this relationship between marriage and poor self-care has also been noticed in other studies [14] [22], this is heterogenous across different settings and study groups, with some showing more favorable results [13] [16] [23].

Better dietary habits amongst rural inhabitants/poorer cohorts within urban 
settings seem to be a noticeable trend in LMICs: urban residents are more likely to be exposed to a westernized diet, whilst rural inhabitants are more inclined to consume staple foods, including a higher vegetable and fruit intake [16] [24]. Illiteracy was also associated with better dietary practices, owing to the fact that many of those living in rural areas tend to be less literate. Noteworthy, self-reported measures as used in this study are subject to patient perception of what constitutes a "good diet": we do not have accurate data on the diet composition to correlate them with the patients' ratings. This could provide valuable insight in future research.

Foot-care practices were the second most frequently observed behavior amongst participants in this study (though still low with only $40.2 \%$ of participants reporting the practice at least 5 days per week). Factors related to good foot-care practice were previous hospital admission related to diabetes, 3 or more hospital visits in the last year, and one or more medical visits during the last month. During such contacts with the healthcare system and providers, participants may have received advice or further information about foot-care: exposure to previous education in other settings has been shown to be associated with frequency of foot care practices [25] [26]. In multivariate analysis, female participants tended to report lower frequency of good foot-care practice, contrary to that noted in other settings [26] [27].

The only factor related to good exercise habits in univariate analysis was living in a rural area, which is not surprising in this context given that rural inhabitants tend to have more physically active jobs and lifestyle. Building a more comprehensive picture of this, multivariate analysis showed that in addition to residing in rural areas, being male and within a low income bracket was also associated with better physical activity, which has also been observed in previous studies [16]. Again, this is not surprising here given the occupational status amongst these residents who are mostly illiterate and would exercise manual jobs of predominantly low income. With regard to the assessment of physical activity based on self-report, there is possible confusion between exercise and activity performed during work or housekeeping, which should be taken into account when interpreting those results.

Within this study, extremely poor levels of BST were noticed amongst all participants with average monitoring being less than 1 day a week (mean $=0.81$ ). The two factors associated with BST here were income and illiteracy: those with higher income reported higher testing frequency, whilst illiterate patients reported lower testing frequency. Whilst some studies that have noted the same observation have concluded this to be a consequence of low education [28], this could also be explained by the cost associated with care for diabetic patients: a recent study by Chadli et al. showed that only $46.8 \%$ of patients performed this testing at home, and just $18 \%$ performed this daily, with cost being highlighted as the biggest barrier to this [10]. Given that blood sugar monitoring equipment is not covered under the scheme offered by the national health system, and with just over $42 \%$ of patients in the present study with health insurance, this could 
be a most prominent barrier restricting improvement of BST practices, as has been noted previously [14] [16] [29] [30]. Moreover, as illiterate patients tend to less access to health information, in conjunction with being more vulnerable to unemployment and poverty, they are at greater risk of facing barriers to access blood sugar monitoring equipment.

Sex appears to be an important determinant of self-care practices, consistent with other studies [16] [28]. However, this finding may be explained by other factors which were explored (such as educational level, professional activity and income). This could also be a consequence of other variables, such as health literacy, empowerment and self-efficacy, that were not fully explored in this study [31]. Socioeconomic status is also an important factor which warrants further assessment. Psychological factors were also not assessed in this study, but they have been shown as important predictors of self-care behaviors and may mediate the effect of other factors such as demographic and socioeconomic ones [32] [33] [34] [35]. Thus, future research should consider exploring those factors for a better understanding of the determinants of self-care. Moreover, future research should also include assessment of other dimensions of self-management, such as adherence to treatment. The self-reported nature of the measure used in this study to assess self-care practices needs to be taken into account while interpreting this data, as they are determined by patients' knowledge and attitudes towards self-management, such as what is considered a "healthful eating plan" and "continuous physical activity". While self-reported measures are of paramount importance in the current conception of health and disease, and are easy to record and to monitor over time, there is a need to assess their correlation with actual practices of patients in the Moroccan context.

\section{Conclusion}

In this paper, we draw attention to a new area of interest for the Moroccan context: patient-reported outcomes and specifically self-care practices in diabetes. We believe that due to increasing trends of the disease globally and in our country, a comprehensive approach to the disease management is needed, taking into account the determinant role of patient engagement in any chronic disease. We also explored associations with some factors we believe are of paramount importance to shape and determine the nature of self-care behaviors among patients here. It is noteworthy, however, that more research is needed in this context in order to better understand the influence of the factors we studied in this analysis on self-care behaviors amongst type 2 DM patients, and to explore additional or intermediate factors, such as the effect of psychological conditions. This understanding should enable the design of appropriate interventions to enhance self-care practices, and better tailor interventions to one area of diabetes management for patients.

\section{Acknowledgements}

The authors would like to thank the participants, the patients' organizations, 
health-care professionals and volunteer interviewers for their valuable contribution in this study.

\section{Conflicts of Interest}

The authors declare no conflicts of interest regarding the publication of this paper.

\section{References}

[1] World Health Organization (2016) Global Report on Diabetes.

[2] WHO (2011) Global Status Report on Non-Communicable Diseases 2010. http://www.who.int/nmh/publications/ncd report2010/en

[3] International Diabetes Federation (2015) IDF Diabetes Atlas. 7.

[4] Bos, M. and Agyemang, C. (2013) Prevalence and Complications of Diabetes Mellitus in Northern Africa, a Systematic Review. BMC Public Health, 13, 387. https://doi.org/10.1186/1471-2458-13-387

[5] Heinrich, E., Schaper, N.C. and Vries, N. (2010) Self-Management Interventions for Type 2 Diabetes: A Systematic Review. European Diabetes Nursing, 7, 71-76. https://doi.org/10.1002/edn.160

[6] Chrvala, C.A., Sherr, D. and Lipman, R.D. (2016) Diabetes Self-Management Education for Adults with Type 2 Diabetes Mellitus: A Systematic Review of the Effect on Glycemic Control. Patient Education and Counseling, 99, 926-943. https://doi.org/10.1016/j.pec.2015.11.003

[7] Schulman-Green, D., Jaser, S.S., Park, C. and Whittemore, R. (2016) A Metasynthesis of Factors Affecting Self-Management of Chronic Illness. Journal of Advanced Nursing, 72, 1469-1489. https://doi.org/10.1111/jan.12902

[8] Gonzalez-Zacarias, A.A., Mavarez-Martinez, A., Arias-Morales, C.E., Stoicea, N. and Rogers, B. (2016) Impact of Demographic, Socioeconomic, and Psychological Factors on Glycemic Self-Management in Adults with Type 2 Diabetes Mellitus. Frontiers in Public Health, 4, 195. https://doi.org/10.3389/fpubh.2016.00195

[9] Lamchahab, F.Z., El Kihal, N., Khoudri, I., Chraibi, A., Hassam, B. and Ait Ourhroui, M. (2011) Factors Influencing the Awareness of Diabetic Foot Risks. Annals of Physical and Rehabilitation Medicine, 54, 359-365.

https://doi.org/10.1016/j.rehab.2011.07.004

[10] Chadli, A., El Aziz, S., El Ansari, N., Ajdi, F., Seqat, M., Latrech, H., et al. (2016) Management of Diabetes in Morocco: Results of the International Diabetes Management Practices Study (IDMPS)-Wave 5. Therapeutic Advances in Endocrinology and Metabolism, 7, 101-109. https://doi.org/10.1177/2042018816643227

[11] Toobert, D.J., Hampson, S.E. and Glasgow, R.E. (2000) The Summary of Diabetes Self-Care Activities Measure: Results from 7 Studies and a Revised Scale. Diabetes Care, 23, 943-950. https://doi.org/10.2337/diacare.23.7.943

[12] Adarmouch, L., Sebbani, M., Elyacoubi, A. and Amine, M. (2016) Psychometric Properties of a Moroccan Version of the Summary of Diabetes Self-Care Activities Measure. Journal of Diabetes Research, 2016, Article ID: 5479216. https://doi.org/10.1155/2016/5479216

[13] Reisi, M., Mostafavi, F., Javadzade, H., Mahaki, B., Tavassoli, E. and Sharifirad, G. (2016) Impact of Health Literacy, Self-Efficacy, and Outcome Expectations on Adherence to Self-Care Behaviors in Iranians with Type 2 Diabetes. Oman Medical 
Journal, 31, 52-59. https://doi.org/10.5001/omj.2016.10

[14] Ashur, S.T., Shah, S.A., Bosseri, S., Fah, T.S. and Shamsuddin, K. (2016) Glycaemic Control Status among Type 2 Diabetic Patients and the Role of Their Diabetes Coping Behaviours: A Clinic-Based Study in Tripoli, Libya. Libyan Journal of Medicine, 11, Article ID: 31086. https://doi.org/10.3402/ljm.v11.31086

[15] Saleh, F., Mumu, S.J., Ara, F., Begum, H.A. and Ali, L. (2012) Knowledge and Self-Care Practices Regarding Diabetes among Newly Diagnosed Type 2 Diabetics in Bangladesh: A Cross-Sectional Study. BMC Public Health, 12, Article No. 1112. https://doi.org/10.1186/1471-2458-12-1112

[16] Gopichandran, V., Lyndon, S., Angel, M.K., Manayalil, B.P., Blessy, K.R., Alex, R.G., et al. (2012) Diabetes Self-Care Activities: A Community-Based Survey in Urban Southern India. The National Medical Journal of India, 25, 14-17.

[17] Rajasekharan, D., Kulkarni, V., Unnikrishnan, B., Kumar, N., Holla, R. and Thapar, R. (2015) Self-Care Activities among Patients with Diabetes Attending a Tertiary Care Hospital in Mangalore Karnataka, India. Annals of Medical and Health Science Research, 5, 59-64. https://doi.org/10.4103/2141-9248.149791

[18] Kassahun, T., Gesesew, H., Mwanri, L. and Eshetie, T. (2016) Diabetes Related Knowledge, Self-Care Behaviours and Adherence to Medications among Diabetic Patients in Southwest Ethiopia: A Cross-Sectional Survey. BMC Endocrine Disorders, 16, 28. https://doi.org/10.1186/s12902-016-0114-x

[19] Hailu, E., Mariam, W.H., Belachew, T. and Birhanu, Z. (2012) Self-Care Practice and Glycaemic Control amongst Adults with Diabetes at the Jimma University Specialized Hospital in South-West Ethiopia: A Cross-Sectional Study. African Journal of Primary Health Care \& Family Medicine, 4, 311. https://doi.org/10.4102/phcfm.v4i1.311

[20] Tan, S.L., Juliana, S. and Sakinah, H. (2011) Dietary Compliance and Its Association with Glycemic Control among Poorly Controlled Type 2 Diabetic Outpatients in Hospital Universiti Sains Malaysia. Malaysian Journal of Nutrition, 17, 287-299.

[21] Tol, A., Shojaeezadeh, D., Eslami, A., Alhani, F., Mohajeritehrani, M., Baghbanian, A., et al. (2012) Evaluation of Self-Care Practices and Relative Components among Type 2 Diabetic Patients. Journal of Education and Health Promotion, 1, 19. https://doi.org/10.4103/2277-9531.99219

[22] Costanian, C., Bennett, K., Hwalla, N., Assaad, S. and Sibai, A.M. (2014) Prevalence, Correlates and Management of Type 2 Diabetes Mellitus in Lebanon: Findings from a National Population-Based Study. Diabetes Research and Clinical Practice, 105, 408-415. https://doi.org/10.1016/j.diabres.2014.06.005

[23] Cosansu, G. and Erdogan, S. (2014) Influence of Psychosocial Factors on Self-Care Behaviors and Glycemic Control in Turkish Patients with Type 2 Diabetes Mellitus. Journal of Transcultural Nursing, 25, 51-59. https://doi.org/10.1177/1043659613504112

[24] El Rhazi, K., Nejjari, C., Zidouh, A., Bakkali, R., Berraho, M. and Barberger Gateau, P. (2011) Prevalence of Obesity and Associated Sociodemographic and Lifestyle Factors in Morocco. Public Health Nutrition, 14, 160-167. https://doi.org/10.1017/S1368980010001825

[25] Mogre, V., Abanga, Z.O., Tzelepis, F., Johnson, N.A. and Paul, C. (2017) Adherence to and Factors Associated with Self-Care Behaviours in Type 2 Diabetes Patients in Ghana. BMC Endocrine Disorders, 17, 20. https://doi.org/10.1186/s12902-017-0169-3

[26] Al Johani, K.A., Kendall, G.E. and Snider, P.D. (2015) Self-Management Practices 
among Type 2 Diabetes Patients Attending Primary Health-Care Centres in Medina, Saudi Arabia. Eastern Mediterranean Health Journal, 21, 621-628. https://doi.org/10.26719/2015.21.9.621

[27] Abdulghani, H.M., AlRajeh, A.S., AlSalman, B.H., AlTurki, L.S., AlNajashi, N.S., Irshad, M., et al. (2018) Prevalence of Diabetic Comorbidities and Knowledge and Practices of Foot Care among Diabetic Patients: A Cross-Sectional Study. Diabetes, Metabolic Syndrome and Obesity: Targets and Therapy, 11, 417-425. https://doi.org/10.2147/DMSO.S171526

[28] Ahmed, M.U., Seriwala, H.M., Danish, S.H., Khan, A.M., Hussain, M., Husain, M., et al. (2016) Knowledge, Attitude, and Self Care Practices amongst Patients with Type 2 Diabetes in Pakistan. Global Journal of Health Science, 8, 1-8. https://doi.org/10.5539/gjhs.v8n7p1

[29] Watkins, Y.J., Quinn, L.T., Ruggiero, L., Quinn, M.T. and Choi, Y.-K. (2013) Spiritual and Religious Beliefs and Practices and Social Support's Relationship to Diabetes Self-Care Activities in African Americans. The Diabetes Educator, 39, 231-239. https://doi.org/10.1177/0145721713475843

[30] Hernandez, R., Ruggiero, L., Riley, B.B., Wang, Y., Chavez, N., Quinn, L.T., et al. (2014) Correlates of Self-Care in Low-Income African American and Latino Patients with Diabetes. Health Psychology, 33, 597-607.

https://doi.org/10.1037/hea0000043

[31] McCleary-Jones, V. (2011) Health Literacy and Its Association with Diabetes Knowledge, Self-Efficacy and Disease Self-Management among African Americans with Diabetes Mellitus. ABNF Journal, 22, 25-32.

[32] Mariye, T., Tasew, H., Teklay, G., Gerensea, H. and Daba, W. (2018) Magnitude of Diabetes Self-Care Practice and Associated Factors among Type Two Adult Diabetic Patients Following at Public Hospitals in Central Zone, Tigray Region, Ethiopia, 2017. BMC Research Notes, 11, 380. https://doi.org/10.1186/s13104-018-3489-0

[33] Schinckus, L., Dangoisse, F., Van den Broucke, S. and Mikolajczak, M. (2018) When Knowing Is Not Enough: Emotional Distress and Depression Reduce the Positive Effects of Health Literacy on Diabetes Self-Management. Patient Education and Counseling, 101, 324-330. https://doi.org/10.1016/j.pec.2017.08.006

[34] Nie, R., Han, Y., Xu, J., Huang, Q. and Mao, J. (2018) Illness Perception, Risk Perception and Health Promotion Self-Care Behaviors among Chinese Patient with Type 2 Diabetes: A Cross-Sectional Survey. Applied Nursing Research, 39, 89-96. https://doi.org/10.1016/j.apnr.2017.11.010

[35] Cheng, L.S., Aagaard-Hansen, J., Mustapha, F.I. and Bjerre-Christensen, U. (2018) Malaysian Diabetes Patients' Perceptions, Attitudes and Practices in Relation to Self-Care and Encounters with Primary Health Care Providers. Malaysian Journal of Medical Research, 2, 1-10. 